\title{
Interações entre crianças hospitalizadas e uma psicóloga, durante atendimento psicopedagógico em enfermaria de pediatria ${ }^{\mathbf{1}}$
}

\author{
Stephânia Cottorello Vitorino \\ Faculdade São Luis \\ Maria Beatriz Martins Linhares \\ Maria Regina Fonseca Lindenberg Minardi \\ Universidade de São Paulo, Ribeirão Preto, SP
}

\begin{abstract}
Resumo
O objetivo do presente estudo foi analisar a interação entre crianças hospitalizadas e uma psicóloga em contexto de atendimento psicopedagógico. Foram realizadas 22 observações do atendimento promovido por uma psicóloga com crianças internadas em enfermaria de pediatria, no período de sete meses. Participaram do estudo 102 crianças com idade mediana na fase escolar. As verbalizações foram gravadas e tanto os comportamentos motores, quanto as atividades realizadas foram registradas. Os resultados revelaram que a psicóloga iniciava mais contatos interativos do que as crianças e atuava predominantemente estimulando a interação entre elas. Os temas sobre as atividades psicopedagógicas ocorreram em maior porcentagem em relação aos demais conteúdos verbais. Verificou-se que a psicóloga envolvia a criança nas atividades realizadas, informando, orientando ou fazendo comentários. As verbalizações sobre hospitalização apresentaram baixa ocorrência. As crianças, apesar de enfermas e hospitalizadas, participaram ativamente e interagiram em situações lúdico-pedagógicas. Os achados mostram a relevância desse tipo de atividades para promoção do desenvolvimento da criança no contexto hospitalar.
\end{abstract}

Palavras-chave: criança hospitalizada; interação; psicopedagogia

\begin{abstract}
Interactions between hospitalized children and psychologist during psycho-educational attendance at a pediatric ward. The aim of the present study was to analyze the interactions between hospitalized children and a psychologist at psycho-educational attendance. Twenty two observations were done about the attendance promoted by a psychologist with children at pediatric ward, during a period of seven months. One hundred and two children participated in this study. The verbalizations were recorded; motor behavior and performed activities were registered. The results showed that the psychologist began more interactions than children and promoted stimulation for interactions between them. The verbalizations about psycho-educational activities were more frequent than other ones. The psychologist involved the child in the activities, instructing, giving orientations and talking about it. Low percentage of verbalizations about hospitalization was found. In spite of children being sick and hospitalized, they participated actively and interacted in playing and learning situations. These findings show the relevance of this kind of activities for promoting the children's development in the hospital context.
\end{abstract}

Keywords: hospitalized children; interaction; psycho-educational attendance

A promoção do desenvolvimento sadio e pleno da criança requer necessariamente que se garantam condições adequadas, possibilitando oportunidades de brincar e de aprender. Esses dois aspectos são amplamente discutidos na teoria sócio-construtivista de Vygotsky (1989), que trata da contínua interação entre as condições sociais e a base biológica do comportamento humano. Para estudar a natureza social do desenvolvimento, dois conceitos funda- mentais foram formulados por Vygotsky (1989), a saber: internalização e zona de desenvolvimento proximal. Esses conceitos relacionam-se com a gênese dos processos mentais superiores, ou seja, a formação das funções psicológicas complexas construídas socialmente ao longo do desenvolvimento humano.

Ao discutir o conceito de zona de desenvolvimento proximal, Vygotsky destaca a importância do brinquedo e do 
aprendizado formal como fatores determinantes do desenvolvimento e aprendizagem da criança. Ao brincar e aprender, a criança elabora e internaliza habilidades e conhecimentos socialmente disponíveis. A atividade que a criança realiza com os outros, incluindo os pais, os professores ou outras crianças, influencia sobremaneira as tarefas evolutivas. Segundo Vygotsky, a relação entre a criança e os objetos se dá por meio de outra pessoa (Coll, Palacios, \& Marchesi, 1996).

A afirmação de que "a criança fará sozinha amanhã, o que hoje realiza em cooperação com o outro" sugere o entrelaçamento entre desenvolvimento e aprendizagem e a relevância das interações sociais como propulsoras da autonomia do indivíduo. O conceito de mediação social postulado por Vygotsky (1989) foi posteriormente retomado por Feuerstein ao tratar da experiência de aprendizagem mediada na teoria da Modificabilidade Estrutural Cognitiva (Feuerstein, Rand, Hoffman, \& Miller, 1980).

Algumas instituições são reconhecidamente responsáveis pelo oferecimento de oportunidades educacionais, envolvendo atividades relacionadas a brincadeiras e aprendizado, tais como: família, creches ou escolas. Porém, muitas vezes, as crianças se deparam com condições restritivas ao seu desenvolvimento, sendo a enfermidade crônica e a conseqüente hospitalização algumas destas condições. Segundo Perrin e Gerrity (1984), cerca de 5 a 10\% de todas as crianças apresentam, em alguma época da infância, uma enfermidade prolongada ou incapacitação moderada ou grave. Essas crianças encontram-se sob condições de risco, tanto do ponto de vista orgânico, quanto nos aspectos emocional e educacional no curso do seu desenvolvimento.

Quando a enfermidade e a hospitalização atravessam a trajetória de desenvolvimento da criança, emergem algumas questões. Qual o impacto da enfermidade e da hospitalização sobre seu desenvolvimento? Como ficam as oportunidades de brincar e aprender da criança? O que a instituição hospitalar tem promovido no sentido de garantir essas oportunidades para a criança internada?

Segundo Gallar (1998), a hospitalização pode acarretar à criança alguns problemas no desenvolvimento, de natureza emocional (ansiedade, depressão), cognitiva (dificuldades de aprendizagem) e motivacional (auto-estima negativa). Cada indivíduo pode reagir de diferentes formas frente à hospitalização, dependendo de sua personalidade, situação sócio-familiar, tipo de enfermidade e atribuição de significado que ele realiza. A enfermidade afeta as interações da criança com o ambiente físico e social em que vive e, por sua vez, os aspectos do ambiente são alterados como conseqüência da enfermidade.

A enfermidade crônica constitui-se em condição de vulnerabilidade da criança, agravada pelas adversidades decorrentes da própria enfermidade, do tratamento e da hospitalização. Trata-se, portanto, de situação de múltiplo risco, que ameaça o desenvolvimento da criança. Porém, a vulnerabilidade da criança e os fatores de risco podem ter seus efeitos negativos atenuados por meio da ativação de recursos da própria criança e de mecanismos de proteção advindos do ambiente, como salientam Yunes e Szymanski (2001). Ao se promover o desenvolvimento da criança através do brincar e do aprender cria-se uma rede de mediação social que pode funcionar como mecanismo protetor para neutralizar as adversidades inerentes à condição de enfermidade e hospitalização. Facilita-se desta forma o desencadeamento do processo de resiliência, que consiste na habilidade de superar o efeito das adversidades e do estresse no curso do desenvolvimento (Yunes \& Szymanski, 2001), através do interjogo entre fatores de risco e proteção; consiste, portanto, na maleabilidade do indivíduo no enfrentamento das situações adversas.

Durante a hospitalização são interrompidas as brincadeiras e a vida escolar da criança, havendo privação da companhia dos familiares e dos companheiros. Os adultos presentes no hospital são, muitas vezes, pessoas estranhas e encontram-se envolvidos em rotinas do tratamento, deixando de atender às necessidades psicológicas da criança. Ela permanece em um ambiente social, em que se estabelecem relações interpessoais, ocorrem novas experiências, porém em condições predominantemente ansiogênicas e imprevisíveis.

Para neutralizar ou eliminar os efeitos adversos da hospitalização aos processos de desenvolvimento e aprendizagem da criança, algumas modalidades de assistência têm surgido com a finalidade de proporcionar experiências de atividades construtivas à criança hospitalizada, tais como: salas de recreação, brinquedotecas, classes hospitalares ou oficina psicopedagógica (Bierman, 1980; Brazelton, 1994; Ceccin \& Fonseca, 1999; Jorge, Mittempergher, \& Ferreti, 1996; Koinsky, 1997; Lindquist, 1993; Linhares \& Minardi, 1999; Mello, Goulart, Ew, Moreira, \& Sperb, 1999; Rae, Worchel, Upchurch, Scanner, \& Daniel, 1989; Ribeiro, 1993; Viegas, 1997).

Com base nessas considerações, o presente estudo teve por objetivo caracterizar indicadores da interação estabelecida entre crianças enfermas hospitalizadas e uma psicóloga, durante o desenvolvimento das atividades de brincar e aprender em contexto de atendimento psicopedagógico em enfermaria de pediatria.

\section{Método}

\section{Participantes}

A amostra de conveniência foi obtida nos períodos de abril a junho e de agosto a novembro de 1998. Foi composta por 102 crianças e adolescentes na faixa etária de 3 a 17 anos, com idade mediana de oito anos, internadas com enfermidades crônicas na Enfermaria de Pediatria do Hospital das Clínicas da Faculdade de Medicina de Ribeirão Preto (Universidade de São Paulo), que freqüentaram o Programa de Apoio Psicopedagógico e Suporte Psicossocial à Criança Hospitalizada. Além das crianças, participou uma psicóloga responsável pelos atendimentos, que possuía especialização em Psicopedagogia. A participação das crianças atendeu aos critérios éticos de pesquisa com seres humanos, tendo sido 
assinado o termo de consentimento livre e esclarecido pelos responsáveis. O estudo foi aprovado pelo Comitê de Ética em Pesquisa do HCFMRP-USP.

\section{Material}

O material utilizado no presente estudo incluiu: (a) protocolo de registro de observação do comportamento motor e das atividades (Vitorino, 2001), em que se anotavam as seguintes informações: sobre as crianças, participantes presentes, início e término da observação, materiais utilizados nas atividades desenvolvidas, comportamentos orientados para a atividade, comportamentos orientados para o contexto fora da atividade, comportamentos orientados para o contexto pessoal, postura, locomoção e posição da criança na sala; sobre a psicóloga, comportamentos orientados para atividade e comportamentos orientados para a criança; (b) ficha de caracterização das crianças e das condições de hospitalização, contendo informações sobre: idade e sexo da criança e clínica pediátrica de origem e enfermidade; (c) prontuários médicos; e (d) gravador e fitas cassete.

\section{Local e contexto da pesquisa}

O estudo foi desenvolvido na sala de recreação da Enfermaria de Pediatria do HCFMRP, em que era realizado diariamente o Programa de Apoio Psicopedagógico e Suporte Psicossocial à Criança Hospitalizada, estruturado com base na perspectiva desenvolvimentista de atendimento psicopedagógico proposta por Linhares (1998). As atividades eram realizadas em grupo, com duas horas de duração, dirigidas a crianças hospitalizadas, tendo por objetivo promover o desenvolvimento psicológico das mesmas.

\section{Procedimento}

\section{Coleta de dados}

Em primeiro lugar, procedeu-se à observação de 22 sessões de atendimento das crianças em grupo, que rotineiramente era realizado pela psicóloga contratada na referida enfermaria. Essas sessões ocorreram ao longo do período de sete meses. Participaram as crianças reunidas em grupos abertos para a realização dos atendimentos psicopedagógicos orientados pela psicóloga. Os grupos foram diversos quanto à sua composição, sendo sua configuração determinada pelas crianças presentes disponíveis na sala. No período, todas as sessões foram incluídas, excetuando-se as sessões sobre datas comemorativas e aquelas em que não havia crianças em grupo. As observações da interação das crianças com a psicóloga foram realizadas de forma sistemática, de acordo com a metodologia proposta por Danna e Matos (1984). O comportamento verbal foi gravado em áudio e os comportamentos motores, as atividades, e seu esquema de realização foram anotados pela pesquisadora (primeira autora), psicopedagoga e treinada em observação sistemática, no protocolo de registro subdividido em intervalos de tempo (referido na seção de material). Em segundo lugar, foram coletadas as informações sobre as características das crianças participantes do estudo nos prontuários médicos, utilizando-se a ficha de caracterização.

\section{Análise de dados}

Primeiramente, realizou-se a transcrição das gravações do comportamento verbal das crianças e da psicóloga no protocolo de registro. Anotava-se o comportamento verbal ao lado do comportamento motor e das atividades registradas, de forma sincronizada por intervalo de tempo. Após a transcrição das sessões de observação dos atendimentos psicopedagógicos, o comportamento verbal foi submetido à análise de conteúdo temático, elaborando-se sistemas de categorias sobre as verbalizações emitidas (Vitorino, 2001). Para tanto, liam-se exaustivamente as verbalizações, extraíam-se as expressões temáticas mais relevantes e estas eram agrupadas em categorias temáticas reunindo conteúdos afins. Tomou-se por base o sistema de categorias verbais, proposto por Linhares e Marturano (1994), para elaborar um sistema de categorias de conteúdo verbal da psicóloga (Tabela 1) e das crianças (Tabela 2).

Em segundo lugar, foi realizado um sistema de categorias sobre os episódios de contato interativo entre as crianças e a psicóloga (Vitorino, 2001), cujas categorias estão descritas a seguir. I - Intercâmbio bidirecional: consiste em iniciativa verbal com resposta de uma ou mais crianças, podendo ser de tipo: (a) foco individual (iniciado pela psicóloga com resposta; iniciado pela criança com resposta; iniciado pelo grupo com resposta da psicóloga; comunicação entre crianças); (b) foco no grupo (iniciado pela psicóloga com resposta individual; iniciado pela psicóloga com resposta do grupo; iniciado pela criança com resposta individual). II - Iniciativa de intercâmbio sem resposta: consiste em tentativa de comunicação com o outro, sem obter resposta, podendo ser de tipo:

Tabela 1

Sistema de categorias de conteúdo das verbalizações da psicóloga

\begin{tabular}{|c|c|}
\hline Categorias & Subcategorias \\
\hline Atividade & $\begin{array}{l}\text { Conduz/Pergunta/Orienta/Informa/Comenta atividade em si. } \\
\text { Informa/Orienta/Pergunta algo decorrente da atividade (Transcendência) } \\
\text { Valoriza o desempenho da criança/Requisita participação } \\
\text { Muda de Atividade/ Comenta Participação }\end{array}$ \\
\hline $\begin{array}{l}\text { Hospitalização } \\
\text { Assunto pessoal }\end{array}$ & $\begin{array}{l}\text { Reconhece sentimentos/ Comenta hospitalização/ Promove solução/ Apoio } \\
\text { Casa/Família }\end{array}$ \\
\hline
\end{tabular}


Tabela 2

Sistema de categorias de conteúdo das verbalizações das crianças

\begin{tabular}{ll}
\hline \multicolumn{1}{c}{ Categorias } & \multicolumn{1}{c}{ Subcategorias } \\
\hline Atividade & Realiza Atividade/Solicita Atividade \\
& Comentários/Perguntas decorrentes do tema da atividade \\
& Recusa/Resiste a participar da atividade \\
& Comentário sobre a atividade \\
Relacionamento com outras crianças & Agressão Verbal/Discussão com outra criança \\
& Comentário negativo sobre outra criança/ Reclama de outra criança \\
& Ordem dirigida à atividade/Ameaça de agressão física \\
& Convite para brincar/Queixa de agressão/ Recusa a brincar \\
& Refere a agressão física \\
& Provoca/Reclama sobre a psicóloga \\
Relacionamento com psicóloga & Queixa/Comentário sobre exame/Tratamento \\
Enfermidade/hospitalização & Enfermidade/hospitalização/Alta \\
Assunto pessoal & Casa/Família/Escola \\
\hline
\end{tabular}

iniciativa da psicóloga de intercâmbio individual, iniciativa da psicóloga de intercâmbio em grupo, iniciativa da criança de intercâmbio com a psicóloga, iniciativa da criança de intercâmbio em grupo, ou iniciativa da criança de intercâmbio com outra criança. III - Intercâmbio cruzado paralelo: consiste em tentativa de comunicação com outro sem que este consiga responder à comunicação, pois há interrupção de um terceiro, que passa a manter comunicação com o primeiro, podendo ser de tipo: intercâmbio entre psicóloga e criança cruzado por intercâmbio de outra criança, ou intercâmbio entre criança e criança/psicóloga/grupo, cruzado por intercâmbio com criança psicóloga. IV - Intercâmbio interrompido: consiste em tentativa de comunicação com o outro sem que se siga uma resposta, devido ao fato de ser interrompido por um terceiro, podendo ser de tipo: intercâmbio entre psicóloga e criança, interrompido por outra criança, intercâmbio entre crianças, interrompido pela psicóloga, intercâmbio entre psicóloga e grupo, interrompido por criança, ou intercâmbio entre criança e psicóloga, interrompido por criança. V - Participação periférica: consiste em tentativa de comunicação com o outro com resposta à comunicação, porém havendo participação de um terceiro indivíduo na comunicação.

O procedimento de análise foi realizado por dois classificadores independentes e as poucas discordâncias foram discutidas e revistas até a obtenção dos sistemas de categorias finais. O índice de acordo foi de 93\%, para as categorias verbais, e $86 \%$, para os episódios de contato interativo. Os dados foram quantificados em termos de freqüência, porcentagem e incidência, dependendo de sua natureza.

\section{Resultados}

A Tabela 3 apresenta os resultados das categorias de direção dos episódios de contato (intercâmbio bidirecional, iniciativa de intercâmbio sem resposta, intercâmbio cruzado paralelo, intercâmbio interrompido e participação periférica), divididos em subcategorias respectivamente, identificados nas sessões de atendimento psicopedagógico. As porcentagens das categorias foram obtidas dividindo-se a freqüência de cada categoria pelo total de ocorrência dos episódios ( $n=$ 3.639). As porcentagens das subcategorias foram obtidas dividindo-se a freqüência de cada subcategoria pelo total das respectivas categorias de episódio de contato. Na descrição das tabelas a letra $\mathrm{P}$ corresponde à psicóloga, $\mathrm{C}$ às crianças $\mathrm{e}$ $\mathrm{G}$ ao grupo de crianças.

Na Tabela 3 pode-se observar que as duas categorias que revelaram as maiores porcentagens, reunindo respectivamente cerca da metade dos episódios de contato, foram: iniciativa de intercâmbio sem resposta (50\%) e intercâmbio bidirecional (47\%). O restante dos episódios de interação (intercâmbio cruzado paralelo, intercâmbio interrompido e participação periférica) apresentou baixa porcentagem, inferior a 0,2\%. Na categoria de iniciativa de intercâmbio sem resposta, observa-se que a psicóloga realizou acentuadamente mais iniciativas de intercâmbio do que as crianças ( $\mathrm{P}=82 \%$; $\mathrm{C}=$ 18\%). Nas iniciativas de intercâmbio da psicóloga predominaram mais as dirigidas às crianças individualmente do que as dirigidas ao grupo.

Na categoria de intercâmbio bidirecional, verifica-se que os intercâmbios foram mais individualizados do que em grupo (individual $=77 \%$; em grupo $=19 \%$ ). Tanto os intercâmbios individuais quanto os em grupo, foram iniciados mais vezes pela psicóloga do que pela criança. No primeiro, a psicóloga iniciava o intercâmbio bidirecional duas vezes mais do que a criança. Observa-se que mesmo quando a psicóloga se dirigia ao grupo, as respostas na sua maioria eram dadas por uma única criança.

A Tabela 4 apresenta os resultados das categorias de conteúdo das verbalizações das crianças emitidas durante as sessões de atendimento psicopedagógico (atividade, relacionamento com criança, relacionamento com psicóloga, enfermidade ou hospitalização e assunto pessoal), divididas em subcategorias, respectivamente. As porcentagens das categorias foram obtidas dividindo-se a freqüência de cada categoria pelo total de ocorrência das verbalizações das crianças ( $n=2.094)$. As porcentagens das subcategorias foram obtidas dividindo-se a freqüência de cada subcategoria pelo total de ocorrência da respectiva categoria verbal da criança. 
Tabela 3

Freqüencia e porcentagem das categorias de direção dos episódios de contato durante as sessões de atendimento psicopedagógico

\begin{tabular}{lrc}
\multicolumn{1}{c}{$\quad$ Direção dos Episódios de Contato } & Freq. & \% \\
\hline Intercâmbio Bidirecional & $\mathbf{1 7 1 2}$ & $\mathbf{4 7}$ \\
$\quad$ Foco individual & 885 & 52 \\
Iniciado pela psicóloga com resposta & 435 & 25 \\
Iniciado pela criança com resposta & 2 & 0,5 \\
Iniciado pelo grupo com resposta da psicóloga & 53 & 3 \\
Comunicação entre crianças & & \\
$\quad$ Foco grupo & 294 & 17 \\
Iniciado pela psicóloga com resposta individual & 40 & 2 \\
Iniciado pela psicóloga com resposta do grupo & 3 & 0,5 \\
Iniciado pela criança com resposta individual & $\mathbf{1 8 3 5}$ & $\mathbf{5 0}$ \\
Iniciativa de Intercâmbio Sem Resposta & 1067 & 58 \\
Iniciativa da psicóloga de intercâmbio individual & 443 & 24 \\
Iniciativa da psicóloga de intercâmbio em grupo & 140 & 8 \\
Iniciativa da criança de intercâmbio com a psicóloga & 106 & 6 \\
Iniciativa da criança de intercâmbio em grupo & 79 & 4 \\
Iniciativa da criança de intercâmbio com outra criança & $\mathbf{1 4}$ & $\mathbf{0 , 6}$ \\
Intercâmbio Cruzado Paralelo & & \\
Intercâmbio entre psicóloga e criança, cruzado por intercâmbio de & 7 & 50 \\
outra criança & & \\
Intercâmbio entre criança e criança/psicóloga/grupo, cruzado por & 7 & 50 \\
intercâmbio com criança/psicóloga & $\mathbf{2}$ \\
Intercâmbio Interrompido & $\mathbf{6 2}$ & 64 \\
Intercâmbio entre psicóloga e criança, interrompido por criança & 40 & 22 \\
Intercâmbio entre crianças, interrompido pela psicóloga & 14 & 8 \\
Intercâmbio entre psicóloga e grupo, interrompido por criança & 5 & 6 \\
Intercâmbio entre criança e psicóloga, interrompido por criança & 3 & $\mathbf{8}$ \\
Participação Periférica & $\mathbf{8}$ & \\
\hline
\end{tabular}

Tabela 4

Freqüência e porcentagem das categorias de conteúdo das verbalizações das crianças, durante as sessões de atendimento psicopedagógico

\begin{tabular}{lrr}
\hline \multicolumn{1}{c}{ Categorias de conteúdo verbalizadas das crianças } & Freq. & $\%$ \\
\hline Atividade & $\mathbf{1 7 8 8}$ & $\mathbf{8 5}$ \\
Realização da atividade & 1609 & 90 \\
Solicitação sobre a atividade & 36 & 2 \\
Comentário/Pergunta decorrente do tema da atividade (transcendência) & 104 & 6 \\
Recusa/ Resistência a participar da atividade & 19 & 1 \\
Comentário sobre a atividade & 20 & 1 \\
Relacionamento com outras Crianças & $\mathbf{3 5}$ & $\mathbf{2}$ \\
Agressão Verbal & 8 & 23 \\
Discussão com outra criança & 7 & 20 \\
Comentário negativo sobre outra criança & 5 & 14 \\
Reclama /Disputa com o colega & 4 & 11 \\
Ordem dirigida à atividade & 4 & 11 \\
Ameaça agressão física & 2 & 6 \\
Convite para brincar & 1 & 3 \\
Pedido/Solicitação a outra criança & 1 & 3 \\
Queixa de agressão & 1 & 3 \\
Recusa a brincar & 1 & 3 \\
Refere agressão física & 1 & 3 \\
Relacionamento com Psicóloga & $\mathbf{2 1}$ & $\mathbf{1}$ \\
Provoca/Reclama sobre a psicóloga & & \\
Enfermidade / Hospitalização & $\mathbf{4 9}$ & $\mathbf{2}$ \\
Queixa/Comentário sobre o exame/Tratamento/Enfermidade/ Hospitalização/Alta & & \\
Assunto Pessoal & $\mathbf{1 3 1}$ & $\mathbf{7}$ \\
Casa/Família/Escola & & \\
Outros & $\mathbf{7 0}$ & $\mathbf{3}$ \\
\hline
\end{tabular}


A Tabela 4 mostra que as verbalizações sobre as atividades realizadas reuniram a maior parte das verbalizações emitidas pelas crianças durante as sessões de atendimento psicopedagógico (85\%). O restante das verbalizações (15\%) foi distribuído predominantemente entre verbalizações sobre assunto pessoal ou relacionamento interpessoal.

A Tabela 5 apresenta os resultados das categorias de conteúdo das verbalizações da psicóloga emitidas durante as sessões de atendimento psicopedagógico (atividade, relacionamento das crianças, hospitalização e assunto pessoal) divididas em subcategorias respectivamente. As porcentagens das categorias foram obtidas dividindo-se a freqüiência de cada categoria pelo total das verbalizações da psicóloga ( $n=3.454)$. As porcentagens das subcategorias foram obtidas dividindo-se a freqüência de cada subcategoria pelo total de ocorrência das respectivas verbalizações da psicóloga.

Observa-se, na Tabela 5, que, de forma semelhante às verbalizações da psicóloga, as verbalizações das crianças sobre as atividades reuniram a maior parte das verbalizações emitidas durante as sessões de atendimento psicopedagógico.

A Tabela 6 apresenta os resultados das atividades desenvolvidas durante as sessões de atendimento psicopedagógico. Foram calculadas as incidências dos diferentes tipos de atividades em grupo e individuais, respec-

Tabela 5

Freqüência e porcentagem das categorias de conteúdo das verbalizações da psicóloga, durante as sessões de atendimento psicopedagógico

\begin{tabular}{lrr}
\multicolumn{1}{c}{ Categorias de conteúdo verbal da psicóloga } & Freq. & $\%$ \\
\hline Atividade & $\mathbf{3 2 1 5}$ & $\mathbf{9 3}$ \\
Conduz/Pergunta/Orienta /Informa/Comenta sobre atividade em si & 2802 & 87 \\
Informa/Orienta/Pergunta algo decorrente da atividade (transcendência) & 170 & 5 \\
Valoriza o desempenho da criança & 127 \\
Propõe atividade & 67 \\
Requisita participação & 25 \\
Muda de atividade & 13 \\
Comenta participação & 11 \\
Relacionamento das Crianças & $\mathbf{3 3}$ \\
Promove interação/Media conflitos & 0,5 \\
Hospitalização & $\mathbf{3}$ \\
Reconhece sentimentos/Comenta hospitalização/Propõe solução/Apoio & $\mathbf{9 0}$ \\
Assunto Pessoal & $\mathbf{1}$ \\
Casa/Família & $\mathbf{1 0 7}$ \\
Outros & $\mathbf{2 , 7}$ \\
\hline
\end{tabular}

Tabela 6

Freqüência e incidência das atividades desenvolvidas em grupo e individualmente nas sessões de atendimento psicopedagógico

\begin{tabular}{lcccc}
\hline \multirow{2}{*}{ Atividades ( $\mathrm{n}=22$ sessões) } & \multicolumn{2}{c}{ Em grupo } & \multicolumn{2}{c}{ Individual } \\
\cline { 2 - 5 } & Freq. & Incid. & Freq. & Incid. \\
\hline Identificação do dia e mês do calendário & 21 & 0,95 & 4 & 0,18 \\
Jogos & 18 & 0,82 & 1 & 0,04 \\
Leitura do livro (narrado ou lido) & 13 & 0,59 & 1 & 0,04 \\
Dobradura & 9 & 0,41 & 0 & 0 \\
Interpretação de história & 8 & 0,36 & 0 & 0 \\
Confecções de trabalho com sucata & 6 & 0,27 & 0 & 0 \\
Dramatizações & 3 & 0,14 & 0 & 0 \\
Desenhos & 1 & 0,04 & 0 & 0 \\
Filme & 1 & 0,04 & 0 & 0 \\
Outra atividade & 1 & 0,04 & 0 & 0 \\
\hline
\end{tabular}


tivamente, dividindo-se a freqüência de ocorrência de cada atividade pelo total de 22 sessões de atendimento psicopedagógico.

Na Tabela 6 observa-se que a maior parte das atividades foi desenvolvida pela psicóloga com as crianças em grupo, mais do que de forma individualizada. Nas atividades em grupo, predominaram, em ordem de maior incidência: identificação do dia e mês do calendário, jogos, leitura de livro de histórias infantis, dobradura e interpretação de histórias.

Na Tabela 7 são apresentados os tipos de episódios de contato, relacionados às verbalizações da psicóloga.

Observa-se na Tabela 7 que quando a psicóloga estabelecia intercâmbios bidirecionais com as crianças individualmente ou em grupo (com ou sem resposta), ela predominantemente verbalizava conduzindo, perguntando, orientando, informando ou comentando sobre a atividade em si. Além disso, quando a psicóloga interagia com o grupo de crianças (com ou sem resposta), ela também verbalizava informando, orientando ou perguntando sobre algo da atividade; desta forma realizava a transcendência do contexto imediato da atividade para outros contextos. As iniciativas da psicóloga para estabelecer intercâmbio com a criança e que não obtiveram resposta da mesma envolviam predominantemente verbalizações relacionadas a: valorização do desempenho da criança na atividade, informação, orientação ou pergunta algo sobre decorrente da atividade como forma de transcendência do contexto imediato e solicitação da participação da criança na atividade. Quando a criança iniciava o intercâmbio bidirecional com a psicóloga, esta verbalizava predominantemente sobre a realização da atividade pela criança e assuntos pessoais sobre casa, família ou escola.

Na Tabela 8 encontram-se os tipos de contato relacionados às verbalizações da criança. Serão considerados apenas os episódios de contato com porcentagem de ocorrência acima de $20 \%$ do total de episódios.

A Tabela 8 revela que quando a psicóloga estabelecia intercâmbio bidirecional com as crianças ou iniciativas de contato em grupo, as crianças verbalizavam predominantemente sobre a realização da atividade ou assuntos pessoais sobre casa, família ou escola. Nas iniciativas de contatos

Tabela 7

Freqüência e porcentagem das categorias de direção dos episódios de contato relacionadas às verbalizações da psicóloga nas sessões de atendimento psicopedagógico

\begin{tabular}{|c|c|c|c|}
\hline Episódios de contato & Freq. & Verbalizações da psicóloga & $\%$ \\
\hline $\begin{array}{l}\text { Intercâmbio bidirecional iniciado } \\
\text { pela psicóloga }\end{array}$ & 885 & $\begin{array}{l}\text { Conduz/Pergunta/ Orienta/Informa/Comenta sobre atividade em si } \\
\text { Assunto pessoal } \\
\text { Informa/Orienta/Pergunta algo decorrente da atividade (transcendência) } \\
\text { Comenta sobre hospitalização }\end{array}$ & $\begin{array}{r}60 \\
18 \\
17 \\
5\end{array}$ \\
\hline $\begin{array}{l}\text { Iniciativa da psicóloga de intercâm- } \\
\text { bio individual (sem resposta) }\end{array}$ & 1067 & $\begin{array}{l}\text { Valoriza desempenho da criança } \\
\text { Informa/Orienta/Pergunta algo decorrente da atividade (transcendência) } \\
\text { Requisita participação } \\
\text { Assunto pessoal } \\
\text { Promove interação } \\
\text { Propõe atividade } \\
\text { Comenta sobre hospitalização }\end{array}$ & $\begin{array}{r}38 \\
32 \\
29 \\
10 \\
7 \\
6 \\
5\end{array}$ \\
\hline
\end{tabular}

Intercâmbio bidirecional com o grupo iniciado pela psicóloga (com resposta individual da criança)

Conduz/Pergunta/Orienta/Informa/Comenta sobre atividade em si

Conduz/Pergunta/Orienta/Informa/Comenta sobre atividade em si

Iniciativa da psicóloga de intercâmbio com o grupo (sem resposta)

Corma/Orienta/Pe

Valoriza o desempenho da criança

Realiza Atividade

Intercâmbio bidirecional iniciado pela criança 
Tabela 8

Freqüência e porcentagem das categorias de direção dos episódios relacionadas às verbalizações da criança nas sessões de atendimento psicopedagógico

\begin{tabular}{|c|c|c|c|}
\hline Episódios de contato & Freq. & Verbalizações da criança & $\%$ \\
\hline \multirow[t]{4}{*}{$\begin{array}{l}\text { Intercâmbio bidirecional iniciado } \\
\text { pela psicóloga }\end{array}$} & 885 & Realização da atividade & 46 \\
\hline & & Assunto pessoal & 36 \\
\hline & & $\begin{array}{l}\text { Comentários/Perguntas decorrentes do tema da } \\
\text { atividade (transcendência) }\end{array}$ & 5 \\
\hline & & $\begin{array}{l}\text { Queixa/Comentário sobre exame/tratamento/ } \\
\text { enfermidade/ hospitalização/alta }\end{array}$ & 5 \\
\hline
\end{tabular}

individuais da psicóloga sem resposta das crianças, estas verbalizavam sobre a realização da atividade e sobre comentários ou perguntas decorrentes do tema da atividade, transcendendo o contexto imediato para outros contextos. Nos intercâmbios iniciados pelas crianças, por sua vez, predominaram as verbalizações da criança sobre a realização das atividades.

\section{Discussão}

Com relação à análise da interação entre crianças e a psicóloga, verificou-se que a metade dos episódios de contato caracterizou-se por iniciativas sem resposta e cerca da outra metade foram intercâmbios bidirecionais, em que as iniciativas de contato eram respondidas pelo outro. Tanto em um tipo de episódio de contato quanto em outro, a psicóloga iniciava mais os contatos do que a criança.

Nos intercâmbios sem resposta, quase a totalidade dos episódios de contato foi iniciado pela psicóloga, sejam dirigidos à criança individualmente, seja em grupo. Por outro lado, nos intercâmbios bidirecionais, a metade era iniciada pela psicóloga e um quarto era iniciado pelas crianças. Percebe-se que, durante as sessões de atendimento psicopedagógico, a psicóloga atuava predominantemente estimulando a interação com crianças e desempenhando o papel de mediadora do desenvolvimento e da aprendizagem no contexto da enfermaria.

A importância do ambiente provedor de estímulos, assim como do papel ativo do mediador, a fim de promover o avanço do nível de base do desenvolvimento da criança para o nível potencial de desenvolvimento, foi primeiramente preconizada na teoria sócio-construtivista de Vygotsky (1989). Posteriormente, Feuerstein et al. (1980) e Fonseca (1995) definiram o papel do mediador de desenvolvimento de aprendizagem estabelecendo os critérios de experiência de aprendizagem mediada. Atribuem destaque especial para os critérios de intencionalidade em promover desenvolvimento e reciprocidade, responsável por provocar interação bidirecional, ou seja, intercâmbios. Os achados do presente estudo revelaram que, no contexto estruturado do atendimento psicopedagógico, a psicóloga promovia um contexto interativo com intencionalidade no estabelecimento de contato com as crianças e entre elas.

Destaca-se ainda que os intercâmbios eram mais individualizados do que em grupo, em que a psicóloga procurava atender às necessidades de cada criança. Apesar de estarem em grupo, a psicóloga dirigia-se freqüentemente às crianças individualmente, assim como mostrava-se responsiva aos contatos individualizados. Essa modalidade interativa traduz outro critério relevante de mediação de aprendizagem formulado por Feuerstein et al. (1980) que consiste na diferenciação psicológica individual. Este critério pressupõe o atendimento diferencial a necessidades específicas do indivíduo, atendendo à sua individualidade psicológica. Em contexto indiferenciado e estereotipado como o de um hospital, essa modalidade interativa de atendimento individualizado das iniciativas de contato da criança representa uma possibilidade de participação ativa e personalizada da criança na interação com o outro. Verificou-se ainda que foram poucos os episódios de contato interativos paralelos, interrompidos ou com participação periférica das crianças. Isso denota que, na maior parte das interações, as crianças demonstravam respeito em esperar a vez do outro falar, aguardando para obter a atenção da psicóloga. Havia predominantemente um padrão interativo regulado e organizado no grupo, respeitando os contatos individuais em curso estabelecidos entre determinada criança e a psicóloga.

$\mathrm{Na}$ análise das verbalizações da psicóloga nas sessões psicopedagógicas, percebeu-se que os conteúdos temáticos versavam predominantemente sobre as atividades realizadas em comparação aos demais conteúdos verbais. Isso revela o envolvimento da psicóloga com as atividades realizadas, expressando-se predominantemente sobre a interação com a criança, conduzindo com respostas, informando ou comentando sobre a atividade em si.

A psicóloga vinculava as crianças a atividades lúdicas e pedagógicas, ou seja, atividades de brincar e aprender, que se constituíam em experiências necessárias ao desenvolvimento adaptativo e saudável de toda e qualquer criança. Vygotsky (1989) salienta a importância do brincar e aprender como oportunidades relevantes e necessárias ao desen- 
volvimento da criança, na medida em que estas criam zonas de desenvolvimento proximal. A criança, ao brincar e aprender, atua acima da sua capacidade real, potencializando o seu desenvolvimento para níveis mais elevados e complexos de funcionamento.

Verifica-se, portanto, que no contexto interativo do trabalho psicopedagógico, a psicóloga promovia o desenvolvimento e aprendizagem das crianças, criando zonas de desenvolvimento proximal, que configuravam oportunidades para as crianças hospitalizadas de funcionar acima do nível de desenvolvimento alcançado até o momento e ativar funções embrionárias ou potenciais, conforme o conceito de Vygostky (1989). As crianças, por sua vez, na maior parte das atividades psicopedagógicas em grupo, aderiram às propostas feitas pela psicóloga, envolviam-se no desenvolvimento das atividades e buscavam estabelecer interações, verbalizando mais sobre as atividades do que sobre hospitalização ou enfermidade.

A estrutura e o funcionamento do atendimento psicopedagógico observado estavam centrados nas atividades e nas iniciativas da psicóloga. Caracterizava-se, portanto, como um trabalho operativo e situacional. De caráter operativo, na medida em que as interações centravam-se intencionalmente nas atividades que estavam sendo desenvolvidas, estabelecendo-se dessa forma uma relação triangular entre criança-atividade-psicóloga. De caráter situacional, na medida em que o foco principal de vinculação da criança era o contexto imediato e presente de realização de atividades e de interação com as pessoas disponíveis na situação.

Identificou-se na interação da psicóloga com as crianças o papel de suporte instrucional, quando estimulava a criança a realizar atividades promotoras de desenvolvimento. As verbalizações do tipo: "Vamos fazer outra coisa legal? Ler uma historinha?”, demonstram claramente que as propostas de realizações de atividades formuladas pela psicóloga às crianças denotavam entusiasmo e significado no envolvimento da criança na tarefa.

Feuerstein et al. (1980) incluem entre os cinco critérios mais relevantes de mediação significativa de desenvolvimento e aprendizagem, o significado. Este traduz o sentido afetivo positivo e o envolvimento em que o mediador deve atribuir significado às situações de aprendizagem, deve valorizar positivamente o ensinado e motivar o aprendiz. A psicóloga, ao fazer atribuição positiva à situação de brincar e aprender, estimulava as crianças a conceberem o sentido de aprender, da curiosidade e da produtividade. No contexto hospitalar em que a apatia e o isolamento muitas vezes fazem parte das reações comportamentais das crianças, parece ser relevante identificar-se nas crianças estudadas um padrão ativo de comportamento e mediação com significado para compartilhar brincadeiras e aprendizado.

Cabe salientar que a temática sobre a hospitalização apresentou baixa porcentagem de ocorrência nas verbalizações da psicóloga. Quando ocorria, ela reconhecia os sentimentos da criança, comentava sobre a hospitalização, dava apoio sempre que a criança requisitava para tal. Nas verbalizações da psicóloga, os temas relacionados à valorização da criança, reconhecimento de sentimentos, assunto pessoal e hospitalização também estiveram presentes. Quando a psicóloga estava desenvolvendo a atividade junto com a criança, ela preocupava-se em valorizar a participação e o empenho da criança na atividade, como por exemplo: "Olha que jeito legal que o Danilo escreveu patinho feio, com duas canetas”, ou “A pessoa fica com vermes na barriga, muito bem!”.

Quando a criança trazia para a sessão sentimentos tais como medo, saudade e dor, a psicóloga reconhecia esses sentimentos procurando colocar-se empaticamente no lugar da criança. Quando a criança não falava sobre seus assuntos pessoais, a psicóloga observava que algo estava incomodando a criança e procurava incentivá-la a falar sobre o que a estava perturbando. Por exemplo, frente à queixa "Eu quero a minha mãe" manifestada por uma criança, a psicóloga fornecia suporte afetivo com a reflexão de sentimento do tipo: "Você acha ruim ficar sem ela aqui, né?”. Com isso, verifica-se que a psicóloga atuava como rede de apoio psicológico à criança enferma, tanto oferecendo suporte afetivo, quando reconhecia sentimentos e abordava assuntos pessoais, quanto oferecendo suporte instrucional, quando orientava e informava sobre a realização de atividades.

Quanto às atividades desenvolvidas nas sessões de atendimento psicopedagógico, verificou-se que predominaram atividades realizadas com calendário, jogos, leitura de livros infantis e informativos e dobraduras. Através do calendário, a psicóloga proporcionava atividades que situassem as crianças no tempo, visto que as crianças hospitalizadas perdem o referencial de tempo. No seu planejamento diário incluía a atividade de calendário, que procurava situar a criança na realidade do dia a dia. O calendário a situava nos dias de semana, dias do mês e identificação de datas comemorativas nacionais ou pessoais.

A psicóloga, ao promover jogos interativos, estimulava a interação entre as crianças, facilitando o desenvolvimento da sociabilidade. Vygotsky (1989) destaca a importância do brinquedo como fatores determinantes para o desenvolvimento da aprendizagem da criança. Ao brincar, a criança elabora e internaliza habilidades e conhecimentos socialmente disponíveis. Quando jogam, as crianças exercitam tarefas evolutivas sociais importantes, envolvendo realização, sucesso e insucesso, respeito a regras e ao outro. Além disso, Erikson (1976) ressalta que as realizações de atividades conjuntas da criança com seus pares ou companheiros constituem-se em tarefa relevante do desenvolvimento psicossocial da criança.

A psicóloga, ao trabalhar com leitura de livros infantis, fornecia modelo de como realizar a leitura para as crianças que não sabiam ler, orientava a criança iniciante no aprendizado da leitura e estimulava o treino de leitura daquelas que já estavam alfabetizadas. Verificou-se que as leituras incluíam decodificação e interpretação de textos. As leituras eram se- 
guidas por trabalhos de dobraduras e desenhos sobre o material lido.

A leitura de livros de histórias infantis constitui-se em um momento de imaginação oferecido à criança hospitaliza$\mathrm{da}$, que permite que elas transcendam do contexto concreto e imediato da hospitalização, da doença e de pensamentos aversivos para um contexto de imaginação com conteúdos prazerosos. A criança pode transpor-se de um plano concreto para outro plano de idéias e imaginação, criando um espaço mental de fantasia transcendente ao contexto hospitalar e suas implicações aversivas. Quando a psicóloga trabalhava com a leitura de livros informativos, por sua vez, ela permitia à criança a oportunidade de novos conhecimentos e estimulava a curiosidade para obtenção de informação. Nos dados analisados observou-se que, quando a psicóloga contava história, ela se centrava na atividade, envolvendo-se com esta e, portanto, reduzindo o comportamento de responder às interrupções da criança.

Além de promover a transcendência através da leitura de histórias, nas verbalizações da psicóloga foram observados indicadores de transcendência em comentários dirigidos à criança. Estabelecia relações entre verbalizações e atividades da criança e comentários sobre aspectos fora do contexto hospitalar. Por exemplo, ao assistir a um filme sobre a Gincana da Páscoa, a criança comenta que na sua escola tem gincana e a psicóloga responde "Que legal! Você gosta?”, introduzindo uma conversa pessoal sobre contextos diversos ao hospitalar. Feuerstein et al. (1980) mencionam que a transcendência na mediação de aprendizagem possibilita generalizações de situações específicas para contextos mais amplos.

As interações entre as crianças e a psicóloga estabeleceram-se, portanto, em contexto estruturado de promoção do desenvolvimento e aprendizagem, com características de mediação significativa. Rae et al. (1989), em estudo sobre o impacto psicossocial da brincadeira em crianças hospitalizadas, enfatizam que o brincar promove desenvolvimento, contudo não se trata de um brincar sem estruturação e sem objetivos definidos, mas sim acompanhado por instrução e mediação. Nesse sentido, não basta a simples exposição da criança a brinquedos no contexto hospitalar; necessita-se, outrossim, de aprendizagem mediada por profissional especializado em promoção do desenvolvimento da criança. Além disso, verificou-se que o trabalho psicopedagógico realizado com caráter recreativo e educacional estruturava-se de forma semelhante ao estudo descrito por Ribeiro (1993).

Com relação às verbalizações da criança, observou-se que houve uma nítida predominância de temas relacionados às atividades realizadas em relação às demais temáticas. As crianças quase não verbalizaram sobre sua doença ou sua internação, verbalizando preferencialmente sobre as atividades. Isso demonstra que, mesmo enfrentando um quadro estressante motivado por enfermidades que as acometiam, as crianças tinham interesse e participação marcante em realizar atividades de brincar e aprender. $\mathrm{O}$ tema relacionado a assunto pessoal também foi abordado nas verbalizações das crianças, em que essas compartilhavam de assuntos sobre escola e família.
Linhares e Minardi (1999) e Ribeiro (1993) enfatizaram que o ambiente circundante, provedor de estímulos e oportunidades de aprendizagem, é fundamental para o desenvolvimento psicológico da criança hospitalizada. Na medida em que as crianças hospitalizadas tiveram oportunidades de brincar e aprender, mediadas por profissional especializado, apresentaram, apesar de doentes e submetidas a procedimentos médicos freqüentes, um conjunto de comportamentos saudáveis e orientados para o desenvolvimento ativo de atividades propostas de estimulação do desenvolvimento. Linhares e Minardi (1999) afirmam que a criança enferma apresenta recursos e comportamentos saudáveis orientados para o seu desenvolvimento psicológico que precisam ser potencializados. Apesar de hospitalizadas, as crianças brincam e continuam a se desenvolver, como afirma Lindquist (1993).

Finalizando, os achados sugerem que o trabalho psicopedagógico em enfermaria deva atender a três objetivos integrados: recreativo-educacional-terapêutico. Através do brincar, do aprender e da reflexão dos sentimentos da criança e suporte psicossocial focal, o trabalho psicopedagógico deve favorecer o desenvolvimento, a aprendizagem e a adaptação psicossocial da criança enferma e hospitalizada. Para tanto, faz-se necessário o trabalho de profissional especializado em desenvolvimento psicológico e com domínio de técnicas de mediação de aprendizagem e de suporte psicossocial. Deve-se promover não só o desenvolvimento, mas, sobretudo, criar mecanismos de proteção contra adversidades, visando desencadear processos de resiliência na trajetória de desenvolvimento da criança enferma e hospitalizada.

\section{Agradecimentos}

A realização deste estudo contou com o apoio financeiro da Fundação de Apoio ao Ensino, Pesquisa e Assistência do Hospital das Clínicas da Faculdade de Medicina de Ribeirão Preto da Universidade de São Paulo.

\section{Referências}

Biermann, G. (1980). A criança e a hospitalização. Documentos Roche, 3, 83-90. Brazelton, T. B. (1994). Momentos decisivos do desenvolvimento infantil (Trad. J. L. Camargo). São Paulo: Martins Fontes.

Ceccin, R. B., \& Fonseca, E. S. (1999). Classe hospitalar. Revista Integração, 9(21), 31-40.

Coll, C., Palacios, J., \& Marchesi, A. (1996). Desenvolvimento psicológico e educação-psicologia e educação (Vol. 2, Trad. A. M. Alves). Porto Alegre: Artes Médicas.

Danna, M. F., \& Matos. M. A. (1984). Ensinado observação sistemática - uma introdução. São Paulo: Edicon.

Erikson, E. H. (1976). Infância e sociedade (Trad. G. Amado). Rio de Janeiro: Zahar.

Feuerstein, R., Rand, Y., Hoffman, M. B., \& Miller, R. (1980). Instrumental enrichment - an intervention program for cognitive modifiability. Ilinois: Scott Foresmam.

Fonseca, V. (1995). Educação Especial - programa de estimulação precoce. Porto Alegre: Artes Médicas. 
Gallar, M. (1998). Promoción de la salud y apoyo psicológico al paciente. Madri: Paraninfo.

Jorge, A. C., Mittempergher, R., \& Ferretti, V. M. R. (1996). Trabalho psicopedagógico em instituição hospitalar. Revista Psicopedagógica, 15(39), 42-43.

Koinski, R. T. (1997). Educação em enfermarias pediátricas. Ciência Hoje, 23(133), 74-75.

Lindquist, I. (1993). A criança no hospital - terapia pelo brinquedo (Trad. R. Z. Ciltman). São Paulo: Página Aberta.

Linhares, M. B. M. (1998). Atendimento psicopedagógico de crianças em serviço especializado de psicologia infantil na área da saúde mental - uma perspectiva desenvolvimentista. Psicopedagogia, 17(46), 30-35.

Linhares, M. B. M., \& Marturano, E. M. (1994). Interações em classe de primeira série com substituição de professora. Psicologia: Teoria e Pesquisa, 10(1), 111-128.

Linhares, M. B. M., \& Minardi, M. R. F. L. (1999). Criança hospitalizada em enfermaria de Pediatria - aspectos psicológicos. In R. C. Labate (Org.), Caminhando para a assistência integral (pp. 331-338). Ribeirão Preto: Scala.

Mello, C. O., Goulart, C. M. T., Ew, R. A., Moreira, A. M., \& Sperb, T. M. (1999). Brincar no hospital - assunto para discutir e praticar. Psicologia: Teoria e Pesquisa, 15(1), 65-74.
Perrin, E. C., \& Gerrity, P. S. (1984). Desenvolvimento das crianças portadoras de enfermidades crônicas. In R. J. Haggerty (Orgs.), Clínicas pediátricas da América do Norte (pp. 21-34). (Trad. E. B. Cosendey \& R. M. Gesteira). Rio de Janeiro: Interamericana.

Rae, W. A., Worchel. F. F., Upchurch, J., Sanner, J. H., \& Daniel, C. A. (1989). The psychosocial impact of play on hospitalized children. Journal of Pediatric Psychology, 4(14), 617-627.

Ribeiro, M. J. (1993). O atendimento à criança hospitalizada - um estudo sobre serviço recreativo-educacional em enfermaria pediátrica. Dissertação de Mestrado não-publicada, Universidade Estadual de Campinas, São Paulo.

Viegas, D. (1997). Brinquedoteca hospitalar - a experiência de Santo André. In S. M. P. Santos (Org.), Brinquedoteca - o lúdico em diferentes contextos (pp. 101-105). Petrópolis: Vozes.

Vitorino, S. C. (2001). Interações entre crianças hospitalizadas e psicóloga no atendimento psicopedagógico em enfermaria de Pediatria. Dissertação de Mestrado não-publicada, Universidade de São Paulo, Ribeirão Preto.

Vygotsky, L. S. (1989). Formação social da mente (Trad. J. Cipolla Neto, L. S. Menna Barreto \& S. Castro Afeche). São Paulo: Martins Fontes.

Yunes, M. A. M., \& Szymanski, H. (2001). Resiliência - noção, conceitos afins e considerações críticas. In J. Tavares (Org.), Resiliência e Educação (pp. 14-41). São Paulo: Cortez.

${ }^{1}$ O presente estudo consistiu em parte da Dissertação de Mestrado em Psicologia defendida na Faculdade de Filosofia, Ciências e Letras da Universidade de São Paulo, Ribeirão Preto, pela primeira autora, realizada sob orientação da segunda autora.

Stephânia Cottorello Vitorino, mestre em Psicologia pela Faculdade de Filosofia, Ciências e Letras da Universidade de São Paulo, Ribeirão Preto, é coordenadora de Educação Especial na Escola Municipal de Guariba, SP e professora no curso de Pedagogia da Faculdade São Luis. E-mail: stephaniacv@yahoo.com.br Maria Beatriz Martins Linhares, doutora em Psicologia Experimental pela Universidade de São Paulo, é coordenadora do Serviço de Psicologia Pediátrica do Hospital das Clínicas e professora no departamento de Neurologia, Psiquiatria e Psicologia Médica da Faculdade de Medicina da Universidade de São Paulo, em Ribeirão Preto. E-mail: linhares@fmrp.usp.br

Maria Regina Fonseca Lindenberg Minardi, especialista em Psicopedagogia pelo Hospital das Clínicas, em Ribeirão Preto, é psicóloga no Hospital das Clínicas da Faculdade de Medicina da Universidade de São Paulo, em Ribeirão Preto. E-mail: mreginaflm@uol.com.br

Endereço para correspondência: (MBML) Laboratório de Pesquisa em Prevenção de Problemas de Desenvolvimento e Comportamento da Criança (salas 52/53); Avenida Tenente Catão Roxo, 2650; Prédio da Saúde Mental; Faculdade de Medicina de Ribeirão Preto - USP; Campus Universitário Monte Alegre; Ribeirão Preto, SP; CEP 14048-900. 